\title{
Eine neue Adressierungsart: Positionsadressierung bei indexikalischen Angaben und funktionalen Angabezusätzen
}

Herbert Ernst Wiegand, Germanistisches Seminar, Universität Heidelberg, Heidelberg, Bundesrepublik Deutschland (wiegand.h.e.oberurff@t-online.de)

\begin{abstract}
Zusammenfassung: Nachdem die wichtigsten Begrifflichkeiten aus dem metalexikographischen Diskurs über Adressierung in den letzten 25 Jahren kurz in Erinnerung gerufen wurden, wird als neue Adressierungsart die Positionsadressierung von indexikalischen Angaben, wie z.B. Silbentrennungsangaben und Angaben der Abtrennbarkeit bei Partikelverben, sowie die von binnenerweiternden funktionalen Angabezusätzen, wie z.B. Auslassungs- und Versendekennzeichnungen, eingeführt. Weiterhin wird die Hypothese vertreten, dass auch nichttypographische Strukturanzeiger positionsadressiert sind. Die Annahme der Positionsadressierung trägt zum genaueren Verständnis der Informationsgewinnung des Benutzers-in-actu bei. Abschließend wird darauf hingewiesen, dass die Berücksichtigung der Positionsadressierung zur Differenzierung von Artikeltextstrukturen beiträgt, auf deren Trägermenge eine Adressierungsrelation definiert ist.
\end{abstract}

Stichwörter: INDEXIKALISCHE ANGABE, ANGABEADRESSIERUNG, ANGABEZUSATZADRESSIERUNG, POSITIONSADRESSIERUNG

\begin{abstract}
A New Type of Addressing: Positional Addressing at Indexical Items and Functional Item Additions. Following a brief reminder of the most important concepts from the metalexicographic discourse of the past twenty five years, a new type of addressing is introduced. This is the positional addressing of indexical items, e.g. items indicating syllable division and items indicating the detachability of particle verbs as well as that of functional item additions, which expand items internally, e.g. omission and end of verse indicators. The hypothesis is further presented that non-typographical structural indicators are also positionally addressed. The supposition of positional addressing contributes to a more precise understanding of the information retrieval of the active user. Finally it is shown that the consideration of positional addressing contributes to the differentiation of article structures where an addressing relation is defined with regard to their structure-carrying set.
\end{abstract}

Keywords: INDEXICAL ITEM, ITEM ADDITION ADDRESSING, ITEM ADDRESSING, POSITIONAL ADDRESSING

\section{Vorbemerkung zum Adressierungs-Diskurs von 1989-2013}

Der lexikographietheoretische Diskurs zur Adressierung in ein- und zweiLexikos 25 (AFRILEX-reeks/series 25: 2015): 446-460 
sprachigen Printwörterbüchern beginnt mit Hausmann/Wiegand (1989: 349ff.) und reicht derzeit bis Wiegand/Gouws (2013: 273-314). In dem Zeitraum, der zwischen dem Erscheinen dieser beiden Handbuchartikel liegt, wurden zahlreiche Arbeiten publiziert, die die Kenntnisse zur Adressierung erweitert und vertieft haben. $\mathrm{Zu}$ nennen sind hier - neben kleineren Darstellungen (vgl. dazu Wiegand 2011: 109-110) — vor allem die folgenden Beiträge, die in der Reihenfolge ihres Erscheinungstermins aufgeführt werden:

Hausmann/Werner 1991; Gouws 1994; Louw/Gouws 1996; Wiegand 2000; 2002; 2007; 2007a, 2011; Wiegand/Gouws 2013; Wiegand 2014: 421-442.

In rund drei Jahrzehnten wurden Forschungsergebnisse erzielt, die dazu geführt haben, dass man in der Metalexikographie das für große Teile der Lexikographie charakteristische Phänomen der Adressierung inzwischen wohl in den allermeisten Aspekten recht gut verstanden hat. Damit der Leser die Positionsadressierung als eine neue Adressierungsart leicht und angemessen in das gegebene metalexikographische Wissenskorpus einordnen kann, werden zunächst zur Erinnerung im folgenden Abschnitt einige gezielt ausgewählte Wissensausschnitte stark gerafft präsentiert.

\section{Ausgewählte Essentials aus dem Wissenskorpus zur Adressierung}

Nicht alle Texte, die zu einem Wörterbuch gehören, weisen Adressierung auf, sondern nur vollständig oder partiell kondensierte. Das sind vor allem die akzessiven Einträge und bei diesen insbesondere die Wörterbuchartikel. Ein vollständig kondensierter Artikeltext weist auf der Ebene der Wörterbuchform keine natürlichsprachliche Syntax auf, weil er nicht mittels der Grammatik irgendeiner natürlichen Sprache, sondern nach den Instruktionen eines lexikographischen Instruktionsbuches erzeugt wurde. Während bei natürlichsprachlichen Texten besonders die Grammatik der Sprache, in der ein Text geschrieben ist und einige Textbildungsregeln sowie gegebenenfalls einige extratextuelle Sachverhalte dem kompetenten Leser, der sie kennt, ermöglichen, anhand der textuellen Daten sprachliche Informationen als kognitive Gegebenheiten $\mathrm{zu}$ gewinnen, indem er - um nur einen einfachen Sachverhalt zu erwähnen das Prädikat eines Satzes auf dessen Subjekt bezieht, ist die Sachlage für einen Benutzer-in-actu selbst dann, wenn er zu den kundigen Benutzern zählt und somit die Benutzungshinweise gut kennt, völlig anders. Denn ein kondensierter Wörterbuchartikel eines Sprachwörterbuches ist ein semiotisches Artefakt, in dem Teile und Eigenschaften einer oder mehrerer natürlicher Sprachen und/oder gegebenenfalls natürlicher Variäten mit Teilen und Eigenschaften anderer semiotischer Systeme zu einem komplexen Textgebilde mit einem genuin lexikographischen Textformat instruktionenbasiert zusammengefügt sind. Besonders die artikelinternen und gegebenenfalls die artikelexkurrenten Adressierungsbeziehungen muss ein Benutzer-in-actu kennen, wenn er nicht 
nur formbezogene Informationen sucht, die die Rechtschreibung betreffen und die dadurch erhältlich sind, dass er bestimmte Angaben einfach aufmerksam wahrnimmt. Eine Adressierungsbeziehung führt immer von einem adressierten Textsegment zu einer anderen Textgegebenheit, die die Bezugsadresse trägt. Warum hier von Textgegebenheit gesprochen wird, wird später genauer erklärt. Jeder Benutzer-in-actu, der anhand eines Wörterbuchartikels Informationen erhalten möchte, die sich nicht nur auf die Form geschriebener Sprache beziehen, muss artikelinterne Daten korrekt auf andere Daten beziehen gemäß den Adressierungsbeziehungen, die im jeweiligen Wörterbuch gelten. Da es relativ viele Datentypen und Datendistributionstypen gibt, müssen auch zahlreiche Typen von Adressierung unterschieden werden. Dies sei im Folgenden lediglich angedeutet (ausführlich dazu u.a. Wiegand 2011).

Adressierte funktionale Textsegmente sind Angaben und funktionale Angabezusätze. Ob Angabetexte als adressiert zu gelten haben oder nicht, ist derzeit kontrovers. Man unterscheidet demgemäß zwischen Angabeadressierung und Angabezusatzadressierung.

\section{(a) Angabeadressierung}

Die Angabeadressierung kann links- oder rechtsgerichtet sein, je nachdem in welcher Richtung die Bezugsadresse vom Benutzer-in-actu zu suchen ist. Besonders die Angabelinksadressierung kann adjazent oder nichtadjazent sein. Sie ist dann adjazent linksgerichtet, wenn der Bezugsadressenträger unmittelbar links der adressierten Angabe situiert ist, wobei bei der Festlegung der unmittelbaren Nachbarschaft nichttypographische Mikrostrukturanzeiger nicht berücksichtigt werden. Sie ist einfach gedehnt linksgerichtet, wenn zwischen adressierter Angabe und Adressenträger gerade eine elementare Angabe situiert ist, und sie ist mehrfach gedehnt linksgerichtet, wenn die adressierte Angabe vom Adressenträger durch mindestens zwei elementare Angaben getrennt ist. Die Anzahl der elementaren Angaben zwischen einer adressierten Angabe und ihrem Adressenträger liefert das Maß für die Adressenentfernung. Sind es z.B. sieben Angaben, hat die Adressenentfernung den Wert 7. Findet sich eine adressierte Angabe auf einer rechten Wörterbuchseite und ihre Adresse auf einer der nächsten Wörterbuchseiten, so dass die adressierte Angabe und ihre Adresse nicht in ein und demselben Wahrnehmungsraum situiert sind, liegt gebrochene Adressierung vor. Bei der Angabeadressierung wird weiterhin zwischen unbedingter, bedingender und bedingter Adressierung unterschieden. Die beiden letztgenannten Adressierungsarten treten auf, wenn im Wörterbuchgegenstandsbereich, wenn-dann-Beziehungen gegeben sind, die lexikographisch vertextet werden. Eine weitere Unterscheidung ist die zwischen Angabenah- und Angabefernadressierung. Bei der Angabenahadressierung ist die Adressierungsbeziehung innerhalb akzessiver Einträge gegeben, so dass zwischen artikelinterner, außentextinterner, binnentextinterner, umtextinterner, 
einschubinterner und registerinterner Angabenahadressierung unterschieden wird. Bei letzterer sind die adressierte Angabe und der Bezugsadressenträger in zwei verschiedenen akzessiven Einträgen situiert, die zugriffsverschieden sind, weil sie im Zugriffsbereich zweier verschiedener äußeren Zugriffsstrukturen liegen. Die Angabefernadressierung kann artikelexkurrent, binnentext-, umtext- und einschubexkurrent sein. Je nachdem, ob der Adressenträger eine lemmatische oder eine nichtlemmatische Angabe ist, wird zwischen lemmatischer und nichtlemmatischer Adressierung unterschieden. Die artikelinterne lemmatische (adjazente oder nichtadjazente) Linksadressierung ist wohl die am häufigsten auftretende Adressierungsart und gilt daher als der klassische Fall. In kondensierten akzessiven Einträgen, die keine Wörterbuchartikel sind und somit keine Lemmazeichengestaltangabe, sondern einen Eintragseingang, wie z.B. einen Umtexteintragseingang (kurz: Umtexteingang), aufweisen, entspricht der lemmatischen die Eingangsadressierung und der nichtlemmatischen die eingangsexterne Adressierung.

Angabenahadressierung tritt nicht nur innerhalb von akzessiven Einträgen auf, sondern auch innerhalb von funktionalen Artikelteilstrecken, und zwar in Artikelnestern und in Artikelnischen. In diesen sind die Nestsublemmata an das Nesteingangslemma und die Nischensublemmata an das Nischeneingangslemma adressiert; da diese Adressierungsbeziehungen nestartikelbzw. nischenartikelexkurrent sind, ist artikelübergreifende Nest- bzw. Nischeneingangsadressierung gegeben, die mehrere Untertypen aufweist. Es sei darauf aufmerksam gemacht, dass eine Reihe von seltener auftretenden Besonderheiten bei der Angabeadressierung nicht erwähnt wurden.

\section{(b) Angabezusatzadressierung}

Funktionale Angabezusätze sind Textsegmente mit Angabefunktion aber ohne Textkonstituentenstatus. Man unterscheidet die oben erweiternden funktionalen Angabezusätze, die auch lexikographische Superscripte heißen, von den unten erweiternden funktionalen Angabezusätzen, die auch lexikographische Subscripte heißen. Im Unterschied zu den phonetischen Super- und Subscripten, die Teil des Wörterbuchgegenstands sind, gehören die lexikographischen zur Wörterbuchform. Weiterhin werden die binnenerweiternden funktionalen Angabezusätze unterschieden, die entweder glossierend oder nichtglossierend sind. Die glossierenden sind entweder Glossate oder Glossatfolgen, die nichtglossierenden sind u.a. grammtikbezogen, wie z.B. Genus-, Numerus- oder Kasuskennzeichnungen, oder phonetikbezogen, wie z.B. Aussprachekennzeichnungen. Während oben und unten erweiternde funktionale Angabezusätze sowohl intern- als auch externlemmatisch auftreten, finden sich binnenerweiternde funktionale Angabezusätze nur externlemmatisch. Lexikographische Superscripte, wie z.B. der Akut über die Lemmazeichengestaltangabe „b ó h r e n” aus Weigand (1873) sind hinabadressiert; der unten situierte Adressenträger 
(hier ", $\mathrm{O}^{\prime \prime}$ ) ist ein Angabeformsegment. Lexikographische Subscripte, wie z.B. der Unterstrich in "liberal“ (HWDG 1984) sind hinaufadressiert; der oben situierte Adressenträger (hier ", $\mathrm{a}^{\prime \prime}$ ) ist ebenfalls ein Angabeformsegment. Binnenglossate sind grundsätzlich adjazent linksadressiert, es sei denn, sie sind nicht das vordere Glossat in Glossatfolgen, wie z.B. in "schmutzige (unanständige, ordinäre, gemeine) Witze, Bemerkungen machen" (HWDG 1984). Nur das vordere Glossat ist adjazent links, das mittlere und das hintere Glossat sind nichtadjazent linksadressiert. In nichtelementaren Glossaten können die Glossatssegmente glossatintern entweder adjazent links adressiert sein, wie z.B. die Bedeutungsidentifizierungskennzeichnung " $1^{\prime \prime}$ in "Schub ... K e g e $1 \mathrm{n}$ alle neune auf ein S. (mit einem Wurf 1) treffen (HWDG 1984), oder sie sind adjazent rechtsadressiert, wie die verdichtete Synonymidentifizierungskennzeichnung "SYN" in "Ereignis ... den en (SYN 'Dingen 2.2') gefasst entgegensehen“ (DGWDaF 2000). Glossierende Glossatsegmente, wie z.B. „Dingen” sind immer glossatexkurrent linksadressiert.

Während alle kondensierten Wörterbuchartikel einem der Typen von Adressierungskonstellationen zugeordnet werden können, z.B. dem Typ der vollständig lemmatischen mit internlemmatischer Adressierung (vgl. z.B. Wiegand 2011: 194ff), gehören alle erweiterten Glossate zu einem der Typen von glossatzugehöriger Adressierungskonstellationen, z.B. zum Typ der vollständig dreifach gleichgerichteten Glossat- und Glossatsegmentadressierung (vgl. Wiegand (2014: 432ff.).

Gibt es in einem Wörterbuchartikel einen funktionalen Angabezusatz oder mehrere, weist der Artikel hybride Artikeltextstrukturen auf. Lexikographische Super- und Subscripte bewirken, dass die Artikeltextstrukturen architektonisch angereicherte Teilstrukturen aufweisen. Binnenerweiternde funktionale Angabezusätze bewirken, dass die Artikeltextstrukturen binnenerweiterungsbedingte Teilstrukturen aufweisen. Eine Übersicht über hybride Artikeltextstrukturen findet man in Wiegand (2015).

Damit sind wichtige Wissensvoraussetzungen genannt, die gegeben sein müssen, um vollständig zu verstehen, warum die Positionsadressierung eine neue Adressierungsart ist und was unter Positionsadressierung verstanden werden soll.

\section{Positionsadressierung von Angaben und funktionalen Angabezusätzen}

$\mathrm{Zu}$ den allermeisten Angabetypen gehören Angaben, die adressiert sind, wenn sie als Textkonstituenten Teile von Artikeltexten sind. Es gibt sehr wenige Angabetypen, deren zugehörige Angaben niemals adressiert sind. Die beiden prominentesten Angabetypen dieser Art sind der Typ der Lemmazeichengestaltangabe sowie der Typ des Formkommentars. Im Folgenden seien hierzu einige Beispiele betrachtet. 
(1) Scherz, der; -es, -e auf Belustigung berechnete

Äußerung od. Handlung, Spaß: ein gelungener,

harmloser, netter, geistvoller, anzüglicher, grober,

übler S.; zu Scherzen aufgelegt sein; sich (mit

jmdm.) einen schlechten S. erlauben; etw. im S.

(nicht im Ernst, nicht in böser Absicht) sagen

+ S. beiseite (im Ernst)

(= wa $_{1}$ aus HWDG 1984)

In wa $a_{1}$ ist Scherz eine als lemmatische Substantivangabe ausgeprägte elementare Lemmazeichengestaltangabe; sie ist elementar, da sie nicht durch eine Anwendung der Methode der funktional-positionalen Segmentation in mindestens zwei Angaben segmentierbar ist. Sie ist durch keinen funktionalen Angabezusatz erweitert und ist daher auch mit dem Lemma als Element der Trägermenge der alphabetischen Hauptzugriffsstruktur des HWDG formgleich. Ihre Angabefunktion erfüllt sie dadurch, dass mit ihr das Lemmazeichen Scherz erwähnt (oder: genannt) wird; damit ist zugleich die Form für den Nominativ Singular des Substantivs Scherz in orthographisch korrekter Schreibung gegeben, die gemäß der deutschen lexikographischen Tradition die Nennform für ein Substantiv ist, zu dem, wenn es morphologisch vollständig entwickelt ist, insgesamt acht Flexionsformen gehören. Mit der Nennung von Scherz ist zugleich das Thema für wa ${ }_{1}$ gegeben, das damit gemäß dem Mikrostrukturprogramm des HWDG (1984) lexikographisch bearbeitet werden kann. Während alle anderen Angaben in wa ${ }_{1}$ ihre genuine Angabefunktion nur dadurch erfüllen können, dass sie artikelintern entweder lemmatisch oder nichtlemmatisch adressiert sind, so dass in wa ${ }_{1}$ die Adressierungskonstellation der partiell lemmatischen Adressierung gegeben ist, sind der eingeschränkt adressenhomogene nichtelementare Formkommentar sowie die Lemmazeichengestaltangabe nichtadressiert. Die Lemmazeichengestaltangabe ist der Bezugsadressenträger für alle lemmatisch adressierten Angaben in wa ${ }_{1}$. Die Bezugsadresse |Scherz| ist daher in dem Sinne polyfunktional, dass bei ihr mehrere lemmatische Adressierungsbeziehungen enden. In wa ${ }_{1}$ gibt es keine Positionsadressierung, denn kein adressierungsfähiges Textsegment ist an eine textuelle Artikelposition adressiert.

(2) Fi $\mid$ lou < [filu $>$ ] m. 6> Spitzbube, Gauner, Schlau-

kopf, gerissener od. leichtsinniger Mensch [frz.,

"Spitzbube" < engl. fellow "Bursche"]

(= wa 2 aus Wahrig-8DW)

In $w_{2}$ ist die Lemmazeichengestaltangabe „Fi|lou“ durch eine Silbentrennungsangabe binnenerweitert. Silbentrennungsangaben heißen auch Worttrennungsangaben (vgl. Bergenholtz [et al. 1997]. Sie werden auch durch Punkte realisiert, wie in 'Ab•gleich•chung (aus BW 1980) oder durch einen unterbrochenen senkrechten Strich wie in Fẹlisen (aus Hollós 2001). Bei Partikelver- 
ben kann ein senkrechter Strich sowohl als Silbentrennungsangabe als auch als Angabe der Abtrennbarkeit fungieren, wie z.B. in 'ab | strah •len (aus BW 1980).

Im Folgenden wird zunächst die binnenerweiterte Lemmazeichengestaltangabe "Fi $\mid \mathbf{l o u}$ " aus wa 2 betrachtet. Der senkrechte Strich in ",Fi|lou" ist einwandfrei als Angabe zu klassifizieren, deren Angabeform durch ein Angabesymbol realisiert ist, da ",Fi|lou" durch eine Anwendung der funktional-positionalen Segmentation so segmentierbar ist, dass die beiden Silbenangaben "Fi" und "lou sowie die Silbentrennungsangabe "|" gegeben sind. Bisher galt die Hypothese für die Angabeadressierung, dass jede Angabe an eine Angabeadresse adressiert ist, deren Bezugsadressenträger ein Textsegment, und zwar entweder eine Angabe, ein Angabesegment oder ein Angabeformsegment ist. Daher tritt nun die Frage auf: An welche Angabeadresse ist die Silbentrennungsangabe " $\mid$ " in wa ${ }_{1}$ adressiert? An die linkssituierte Adresse |Fi|, deren Adressenträger das Angabeformsegment " $\mathbf{F i}^{\prime \prime}$ ist, oder an die rechtssituierte Adresse |lou|, deren Adressenträger das Angabeformsegment "lou" ist, oder an beide adjazent situierte Adressen? Es ist hier noch einmal expressis verbis daran erinnert, dass ein Benutzer-in-actu um eine lexikographische Information als kognitive Entität zu erhalten, adressierte Angaben auf ihre Angabeadressen beziehen muss. Das ein Benutzer-in-actu die Silbentrennungsangabe "| $\mid$ " aber auf eine der beiden Silbenangaben oder gar auf beide bezieht, um die Information zu erhalten, dass eine Silbentrennung gerade an der Stelle möglich ist, an der die Silbentrennungsangabe steht, ist mehr als kontraintuitiv. Vielmehr ist es wohl doch eher so, dass eine Silbentrennungsangabe, gleichgültig, ob sie durch einen senkrechten Strich, einen unterbrochenen senkrechten Strich oder einen Mittenpunkt (oder: Mittelpunkt) realisiert ist, dem Benutzer die Stelle zeigt, an dem eine Silbentrennung erfolgen darf. Deswegen nenne ich ab jetzt Silbentrennungsangaben und alle Angaben, die auf die gleiche Weise funktionieren, im Anschluss an den Typ des indexikalischen Zeichens indexikalische Angaben, womit zugleich ein bisher nicht bekannter Angabetyp gefunden ist. Für indexikalische Angaben gilt: Sie sind positionsadressiert: Der Benutzer-in-actu muss mithin z.B. die Silbentrennungsangabe "| $\mid$ " in "Fi | lou" auf die textuelle Position beziehen, in der die Silbentrennungsangabe steht, damit er die lexikographische Information erhält, die zu vermitteln der genuine Zweck einer Silbentrennungsangabe ist. Das Gleiche gilt für die Angabe der Abtrennbarkeit bei Partikelverben und für weitere indexikalische Angaben wie beispielsweise die Angabe zur Identifizierung der Wortbildungskonstituenten in den Lemma aber|kauf, aber|könig und ab|ermorden (aus FWB 1989, vgl. dazu Reichmann 1998: 138). Angaben zur Identifizierung der Wortbildungskonstituenten finden sich auch in Lernerwörterbüchern, wie z.B. in Fahrer|er•laub•nis und fahhr•plan | mäßig (aus ${ }^{3} \mathrm{LGWDaF}$ 1993). Bei indexikalischen Angaben führt die Adressierungsbeziehung also nicht zu einem anderen Textsegment, sondern zu einer anderen Textgegebenheit (vgl. oben), nämlich demjenigen textuellen Ort, an dem die indexikalische Angabe steht.

Auch binnenerweiternde funktionale Angabezusätze können positionsadressiert sein, wie z.B. die in eckigen Klammern stehende Auslassungskenn- 
zeichnung $[\ldots]$ in wa3. Die eckigen Klammern gelten als Angabestrukturanzeigerpaar.

(3) abbau, der (daneben noch undurchsichtig gewordenes abau $<\hat{a}-b \hat{u}$; (e) s/-e....

AuBIN, Weist. Köln/Brühl 41, 40 (rib., 15. Jh): sal men [...] ouch wroigen alle abouwe des boefs

(= wa $_{3}$ aus FWB 1989)

Die Auslassungskennzeichen "..." in wa $\mathrm{w}_{3}$ ist ein indexikalischer funktionaler Angabezusatz, der den Ort des inneren Belegtextschnitts kennzeichnet.

(4) äffer, der; $-I-\varnothing$

1. $>$ Gaukler, Scharlatan, Possenreißer $<; \ldots$

2. > Betrüger $<$; zu affen 2.

FASTNACHTSP. 606,9 (nobd.,15. Jh.): Du pist ain rechter trieger | Und ain effer und ain lieger

(wa ${ }_{4}$ aus FWB 1989)

In wa $a_{4}$ ist der senkrechte Strich " $\mid$ “ in der Belegtextangabe ein binnenerweiternder indexikalischer funktionaler Angabezusatz vom Typ der Versendekennzeichnung. Zwei Versendekennzeichnungen mit paarigem Angabestrukturanzeiger können zu einer Versauslassungskennzeichnung " $|[\ldots]|$ " kombiniert werden, wie in:

(5) affenort, das

phraseologisch jn. auf das affenort setzen

$>$ jn. zum Narren halten $<$ mit Öffnung zu

$>$ jn. betrügen $<$

FASTNACHTSP. 855, 9 (nobd., n. 1450); Hort, junger geselle, sie ist mein swester | Und hat mir sere geclagt gestern, $|[\ldots]|$ Du habst ir dick und vil gesworen | [...]| Und hab deinen willen getan an gelt, | Des werde sie nimmermer von dir ersetzt, | Und habst sie auf das affenort gesetzt, SCHLOSSER, H. v. Sachsenh. 1618 (schwäb., 1453): Sie saczt mich uff das offen ort | Und hielt mich als ein jungen goch.

(= wa5 aus FWB 1989)

\section{Sind artikelinterne nichttypographische Strukturanzeiger positions- adressiert?}

Die letzte zusammenfassende Darstellung zu Strukturanzeigern ist meines Wissens ein Abschnitt in Wiegand 2005: 333-337. Alle dort formulierten Ausführungen sind auch derzeit noch gültig. Neu hinzugekommen sind die paarigen nichttypographischen Angabestrukturanzeiger (vgl. Wiegand 2010: 514), die nicht wie die nichttypographischen Mikrostrukturanzeiger den Benutzer dabei unterstützen, die artikelinterne Angabeverteilung leichter zu erkennen, son- 
dern anzeigen, dass eine elementare Angabe eine für den Benutzer-in-actu relevante interne Gliederung aufweist. Solange die Positionsadressierung nicht bekannt war, war nicht zu erwarten, dass eine Frage danach, ob nichttypographische Strukturanzeiger als adressiert zu gelten haben oder nicht, als sinnvoll gelten könnte. Denn an welche Textsegmente sollten z.B. die drei satzzeichenartigen Trennzeichen (sensu Wiegand 2005: 335) im Formkommentar von wa ${ }_{1}$, nämlich das Komma nach der Lemmazeichengestaltangabe "Scherz", das Semikolon nach der Artikelangabe "der" und das Komma nach der verdichteten Singularbildungsangabe "-es" adressiert sein? Die Annahme, dass nichttypographische Strukturanzeiger vom Typ des Trennzeichens entweder adjazent links- oder adjazent rechtsadressiert sind, kann sicherlich nichts dazu beitragen, die Informationsgewinnung eines Benutzer-in-actu genauer $\mathrm{zu}$ erklären. Nimmt man aber an, das nichttypographische Strukturanzeiger, deren genuine Funktion ja wörterbuchformbezogen ist, gerade auf den textuellen Ort zu beziehen sind, an dem sie stehen, weil der Benutzer-in-actu durch sie erfährt, dass links von ihnen ein anderes Formelement steht als rechts von ihnen, dann ist die Annahme, nichttypographische Strukturanzeiger seien als indexikalische Zeichen zu verstehen und damit positionsadressiert eine einleuchtende Hypothese, die bei der Erklärung hilft, wie der Benutzer verschiedene Textsegmente entweder unterscheidet oder zusammenordnet.

\section{Schlussbemerkung: Hinweise zur Differenzierung von Artikelanga- ben- und exhaustiven Artikelangabenstrukturen}

Es ist klar, dass die Berücksichtigung der Positionsadressierung bei der Strukturbildung und Strukturdarstellung von Artikelangabenstrukturen und exhaustiven Artikelangabenstrukturen geringfügige Differenzierungen bewirken. Dies sei an der Darstellung der abstrakten hierarchischen Kernstruktur eines einfachen Formkommentars ( $\left.\mathrm{fk}_{1}\right)$ als Substruktur einer Artikelangabenstruktur und einer exhaustiven Artikelangabenstruktur erläutert, ohne dass der Strukturbildungsprozess eingehend erläutert wird.

$\mathrm{fk}_{1}$ : Hoo |li | gan [' hu:lign], der; -s, -s (aus: Duden-7DUW 2011)

Die exhaustive funktional-positionale Segmentation von $\mathrm{fk}_{1}$ erbringt die folgenden Textsegmente, die bei der nachfolgenden Aufzählung mittels des Symbols " $\in$ " für die Element-Klassenrelation ihren Textsegmentklassen zugeordnet werden.

Hoo $\in$ SA (= Silbenangabe)

$\mid \in \operatorname{StrA}$ (= Silbentrennungsangabe)

$\mathbf{l i} \in \mathrm{SA}$ (= Silbenangabe)

gan $\in$ SA (= Silbenangabe) 
$[\in v Z o Z$ (= vorderes Zusammenordnungszeichen)

"hu:lign" $\in$ AusA (= Ausspracheangabe)

]$\in h Z o Z$ (= hinteres Zusammenordnungszeichen)

, $[=$ Komma] $\in T Z$ (= Trennzeichen)

der; -s, $-\mathrm{s} \in$ MorA.S (= Morphologieangabe bei Substantiven)

der $\in$ ArtA (= Artikelangabe)

-s $\in$ v.SgbA (= verdichtete Singularbildungsangabe)

; [ = Semikolon] $\in T Z$ (= Trennzeichen)

-s $\in$ v.PlbA (= verdichtete Pluralbildungsangabe)

Im Folgenden werden zuerst zwei abstrakte hierarchische linke Kernstrukturen als Substrukturen der zugehörigen Artikelangabenstrukturen gebildet; Es sei daran erinnert, dass Artikelangabenstrukturen um Adressierungsstrukturen erweiterte Artikelmikrostrukturen sind. Bei der Struktur (1) wird die Positionsadressierung nicht, bei der Struktur (2) wird sie dagegen berücksichtigt; beide Strukturen finden sich in Abb. 1. Zunächst wird eine Trägermenge gebildet; sie heiße $M_{\text {liKS }}^{a}\left(\mathrm{fk}_{1}\right)$ und kann wie folgt mit einer Mächtigkeit von $|10|$ notiert werden:

$\mathrm{M}_{\text {liKS }}^{\mathrm{a}}\left(\mathrm{fk}_{1}\right)=\left\{\mathrm{FK}, \mathrm{LZG}(2 \mathrm{STr} \mathrm{A}) \mathrm{A}, \mathrm{SA}, \mathrm{ST}_{1} \mathrm{~A}\right.$, AusA, MorA.S, ArtA, DekKA, v.SgbA, v.PlbA $\}$

Auf $M_{\text {liKs }}^{\mathrm{a}}\left(\mathrm{fk}_{1}\right)$ werden dann drei strukturprägende Relationen definiert: eine Präzedenzrelation, eine partitive Relation und eine Angabeadressierungsrelation. Das Ergebnis dieser Definitionsoperationen ist die Struktur (1) in Abb. 1.

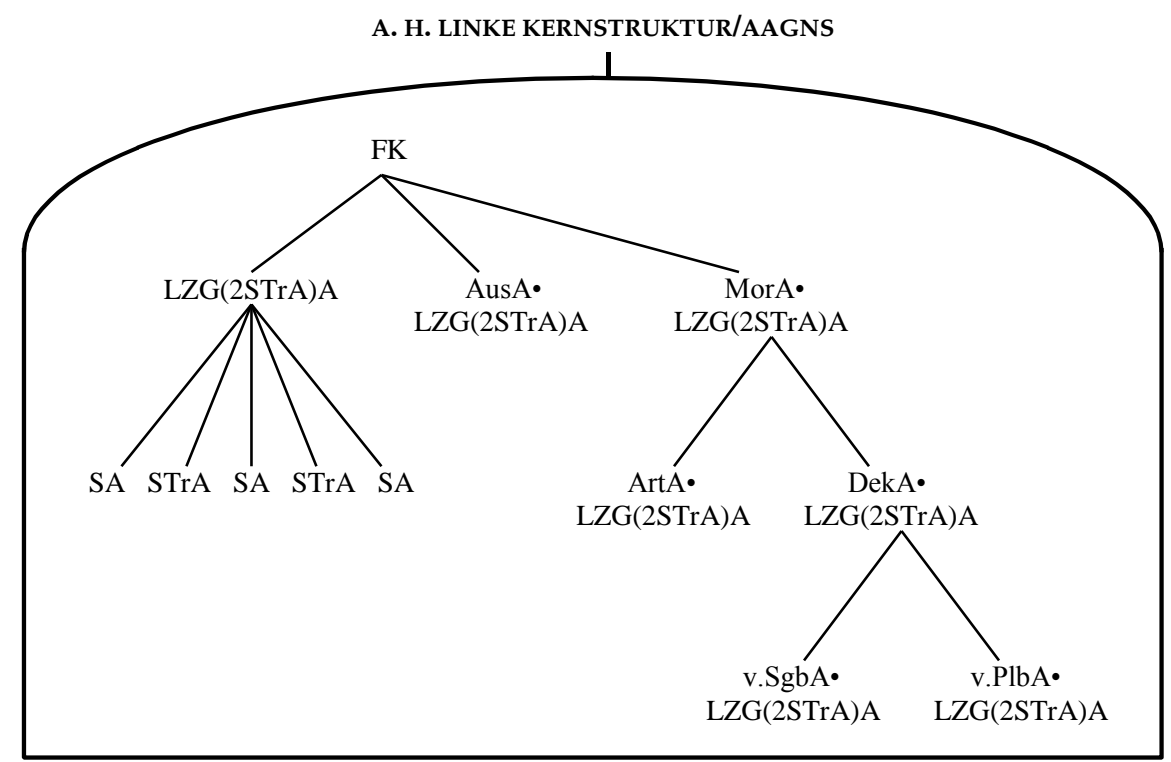




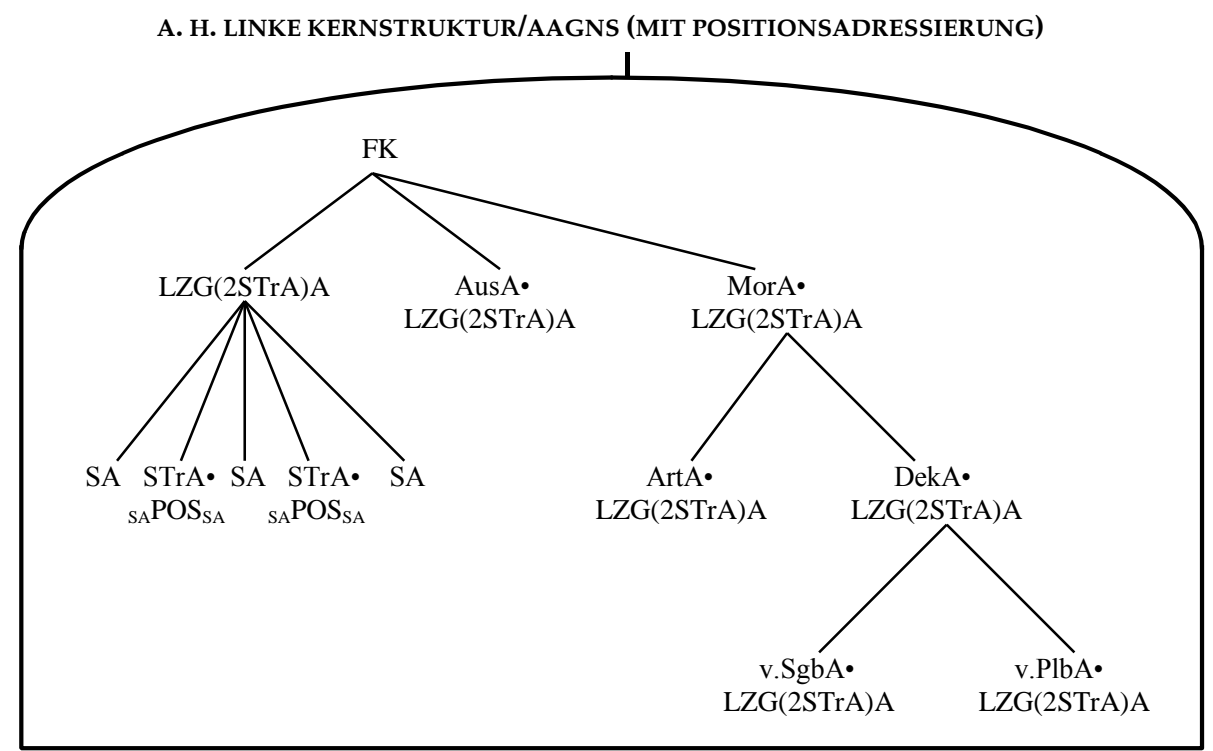

Abb. 1: Strukturgraphe für die abstrakte (a) hierarchische linke Kernstruktur (liKS) als Substruktur der abstrakten hierarchischen Artikelangabenstruktur, die $\mathrm{zu} \mathrm{fk}_{1}$ gehört; /AAGNS ist zu lesen wie als Substruktur der Artikelangabenstruktur; $\mathrm{X} \bullet \mathrm{Y}$ ist zu lesen wie $\mathrm{X}$ ist adressiert an $Y$

Im Folgenden wird die abstrakte hierarchische linke Kernstruktur als Substruktur der abstrakten hierarchischen Artikelangabenstruktur so gebildet, dass die Positionsadressierung der Silbentrennungsangaben Berücksichtigung findet. Dazu muss zunächst die Trägermenge $\mathrm{M}_{\mathrm{liKs}}^{\mathrm{a}}\left(\mathrm{fk}_{1}\right)$ um die Klasse der Positionen zur Trägermenge erw $\mathrm{M}_{\text {liks }}^{\mathrm{a}}\left(\mathrm{fk}_{1}\right)$ erweitert werden. Da die Positionsadressierungsrelation und die Angabeadressierungsrelation zu demselben Relationstyp gehören, nämlich zum Typ der zweistelligen asymmetrischen und irreflexiven Adressierungsrelation, muss lediglich festgelegt werden, dass die Variable " $y$ " im Relationsterm $x$ ist andressiert an $y$ auch mit Positionen (bzw. Klassen von Positionen) belegbar ist. Eine andere Möglichkeit besteht darin, dass man zwei Adressierungsrelationen auf erw $\mathrm{M}_{\mathrm{liKs}}^{\mathrm{a}}\left(\mathrm{fk}_{1}\right)$ definiert, eine Angabe- und eine Positionsadressierungsrelation und die zugehörigen Belegungsvorschriften demgemäß spezifiziert. Als Klassensymbol für adressentragende Positionen wird „POS" gewählt. Das Symbol kann unten indiziert werden (z.B. ${ }_{x} \mathrm{POS}_{\mathrm{y}}$ ), um eine Unterklasse von Positionen zu spezifizieren, z.B. ${ }_{\mathrm{SA}} \mathrm{POS}_{\mathrm{SA}}$ als Symbol für Positionen zwischen zwei Silbenangaben. Definiert man auf erw $\mathrm{M}_{\text {liks }}^{\mathrm{a}}\left(\mathrm{fk}_{1}\right)$ die drei strukturprägenden Relationen, ist das Ergebnis die Struktur (2) in Abb. 1. 
Analog zum bisherigen Vorgehen werden nun zwei abstrakte hierarchische linke Kernstrukturen als Substrukturen der zugehörigen exhaustiven Artikelangabenstrukturen gebildet. Es sei daran erinnert, dass exhaustive Artikelangabenstrukturen erweiterte Artikelkonstituentenstrukturen sind (vgl. z.B. Wiegand 2015), so dass alle nichttypographischen Mikrostrukturanzeiger als Textkonstituenten berücksichtigt werden. Bei der Struktur (1) wird die Positionsadressierung nicht, bei der Struktur (2) wird sie dagegen berücksichtigt. Damit die Struktur (1) erhältlich ist, muss zunächst $M_{\text {liKs }}^{a}\left(\mathrm{fk}_{1}\right)$ um die Klassen der nichttypographischen Mikrostrukturanzeiger $\mathrm{zu}_{\mathrm{liKS}}^{\mathrm{a}}\left(\mathrm{fk}_{1}\right)$ erweitert werden, so dass sie wie folgt mit einer Mächtigkeit von $|13|$ notiert werden kann: $\operatorname{erw}_{1} \mathrm{M}_{\text {liKS }}^{\mathrm{a}}\left(\mathrm{fk}_{1}\right)=\{\mathrm{FK}, \mathrm{LZG}(2 \mathrm{STrA}) \mathrm{A}, \mathrm{SA}, \mathrm{STr} \mathrm{A}$, AusA, MorA.S, ArtA, DekKA, v.SgbA, v.PlbA, TZ, vZoZ, $h Z \circ Z$ \}. Definiert man auf dieser Trägermenge eine Relation vom Typ der Präzedenzrelation, eine vom Typ der partitiven Relation und eine vom Typ der Angabeadressierungsrelation, ist die Struktur (1) in Abb. 2 erhältlich. Die Struktur 2 in Abb. 2 ist erhältlich, wenn man die Trägermenge erw $\mathrm{M}_{1 \text { liKs }}^{\mathrm{a}}\left(\mathrm{fk}_{1}\right)$ um das Symbol POS erweitert und die Symbole für die Klassen von nichttypographischen Strukturanzeigern $v Z$ Z Z, hZoZ und TZ um einen unteren Index $P$ erweitert, der anzeigt, das alle Elemente der Klasse positionsadressiert sind. $T Z_{p}$ beispielsweise ist dann zu lesen wie Klasse der Trennzeichen, die positionsadressiert sind.

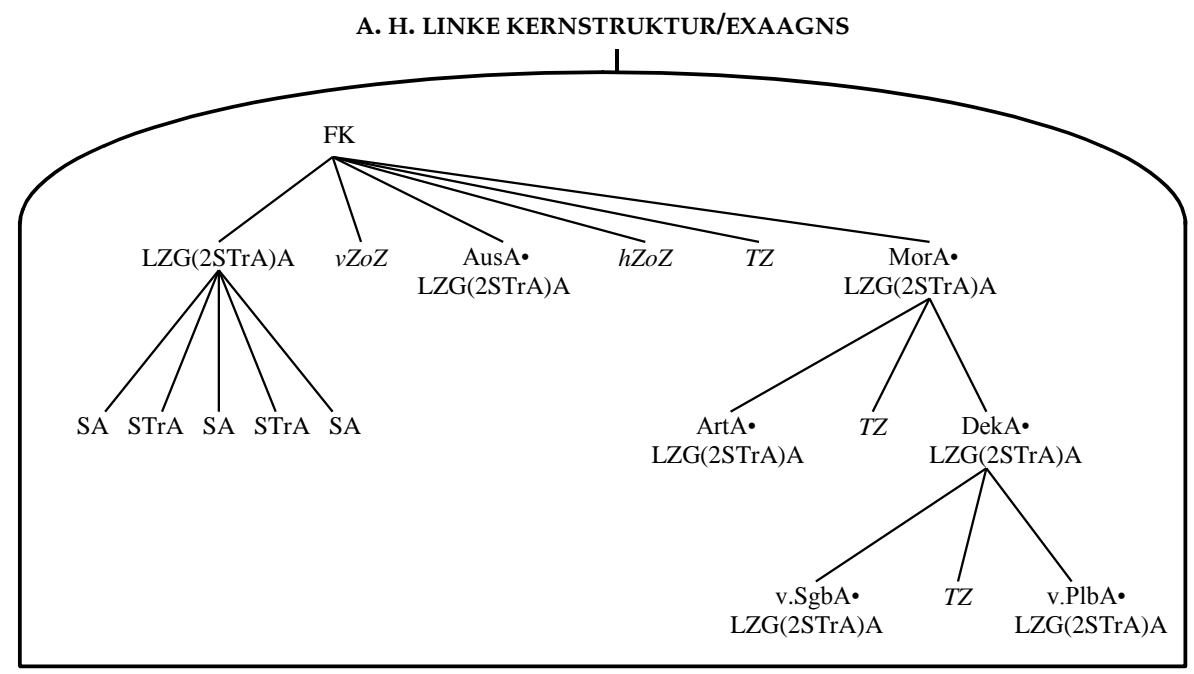




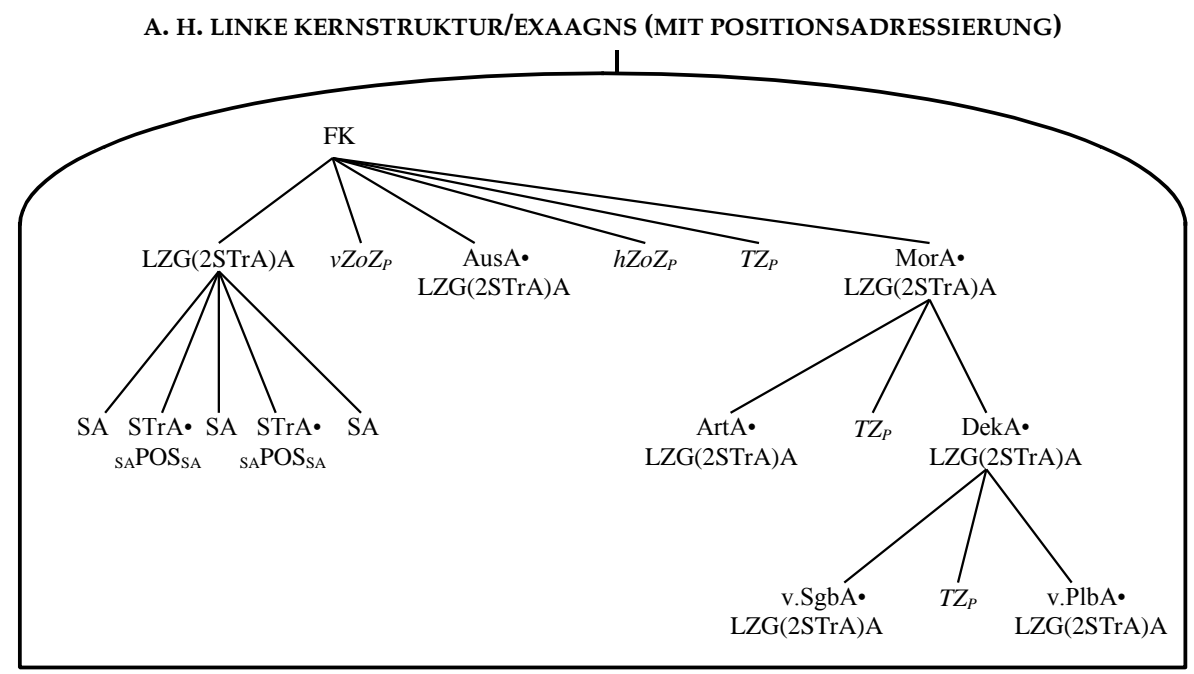

Abb. 2: Strukturgraph für die abstrakte hierarchische linke Kernstruktur als Substruktur der abstrakten hierarchischen exhaustiven Artikelangabenstruktur; /EXAAGNS ist zu lesen wie als Substruktur der exhaustiven Artikelangabenstruktur.

\section{Literatur}

Bergenholtz, Henning, Ilse Cantell, Ruth Vatvedt Fjeld, Dag Gundersen, Jón Hilmar Jónsson und Bo Svensén. 1997. Nordisk Leksikografisk Ordbok ... Oslo: Universitetsforlaget.

BW 1980 = Wahrig, Gerhard, Hildegard Krämer und Harald Zimmermann (Hrsg.). 1980. BrockhausWahrig. Deutsches Wörterbuch in sechs Bänden. 1. Bd. A-BT. Wiesbaden/Stuttgart: F.A. Brockhaus/Dt. Verlagsanstalt.

DGWDaF 2000 = Kempcke, G. (Hrsg.). 2000. De Gruyter Wörterbuch Deutsch als Fremdsprache. Berlin/ New York: Walter de Gruyter.

Duden-7DUW 2011 = Dudenredaktion (Hrsg.). 2011. Duden. Deutsches Universalwörterbuch. 7., überarb. u. erw. Aufl. Mannheim/Zürich: Dudenverlag.

FWB 1989 = Anderson, R.R., U. Goebel und O. Reichmann (Hrsg.). 1989. Frühneuhochdeutsches Wörterbuch. Bd. 1. Einführung a-äpfelkern. Reichmann, O. (Bearb.). Berlin/New York: Walter de Gruyter.

Gouws, Rufus H. 1994. Ostensiewe adressering in vertalende woordeboeke. Lexikos 4: 61-85.

Gouws, Rufus H., Ulrich Heid, Wolfgang Schweickard und Herbert Ernst Wiegand (Hrsg.). 2013. Dictionaries. An International Encyclopedia of Lexicography. Supplementary Volume: Recent Developments with Focus on Electronic and Computational Lexicography. Berlin/Boston: Walter de Gruyter.

Hausmann, Franz Josef, Oskar Reichmann, Herbert Ernst Wiegand und Ladislav Zgusta (Hrsg.). 1989-1991. Wörterbücher. Ein internationales Handbuch zur Lexikographie/Dictionaries. An International Encyclopedia of Lexicography/Dictionnaires. Encyclopédie internationale de lexicographie. 3 Teilbände. Berlin/New York: Walter de Gruyter. 
Hausmann, Franz Josef und Reinhard Otto Werner. 1991. Spezifische Bauteile und Strukturen zweisprachiger Wörterbücher: eine Übersicht. Hausmann, Franz Josef, Oskar Reichmann, Herbert Ernst Wiegand und Ladislav Zgusta (Hrsg.). 1989-1991: 2729-2769.

Hausmann, Franz Josef und Herbert Ernst Wiegand. 1989. Component Parts and Structures of General Monolingual Dictionaries: A Survey. Hausmann, F.J., O. Reichmann, H.E. Wiegand und L. Zgusta (Hrsg.). 1989-1991: 328-360. [Wieder abgedruckt in: Hartmann, R.R.K. (Hrsg.). 2003. Lexicography: Critical Concepts. Vol. III: Lexicography, Metalexicography and Reference Science: 207-254. London/New York: Routledge].

Hollós, Zita. 2001. Német-magyar Suliszótar. Szeged: Grimm Könyv kiadó.

HWDG 1984 = Handwörterbuch der deutschen Gegenwartssprache. 1984. In zwei Bänden. Von einem Autorenkollektiv unter der Leitung von Kempcke, G. [...] Bd. 1: A-K; Bd. 2: L-Z. Berlin: Akademieverlag.

${ }^{3}$ LGWDaF 1993 = Götz, D., G. Haensch und H. Wellmann (Hrsg.). 1993. Langenscheidts Großwörterbuch Deutsch als Fremdsprache. Das neue einsprachige Wörterbuch für Deutschlernende. Berlin [u.a.]: Langenscheidt.

Louw, Phillip Adriaan und Rufus H. Gouws. 1996. Lemmatiese en nielemmatiese adressering in Afrikaanse vertalende Woordeboeke. Suid-Afrikaanse Tydskrif vir Taalkunde 14(3): 92-100.

Reichmann, Oskar. 1989. Lexikographische Einleitung. Anderson, R.R., U. Goebel und O. Reichmann (Hrsg.). 1989. Frühneuhochdeutsches Wörterbuch. Bd. 1. Einführung a-äpfelkern. Reichmann, O. (Bearb.). Berlin/New York: Walter de Gruyter.

Wahrig-8 ${ }^{-}$DW 2006 = Wahrig-Burfeind, Renate (Hrsg.). 2006. Deutsches Wörterbuch. Mit einem Lexikon der Sprachlehre. 8. vollständig neu bearb. Aufl. Gütersloh/München: Wissen Media Verlag $\mathrm{GmbH}$.

Weigand, Friedrich Ludwig Karl. 1873. Deutsches Wörterbuch. 2. verb. u. verm. Aufl. 1. Bd. A-L. Gießen: Ricker.

Wiegand, Herbert Ernst. 2000. Adressierung in der ein- und zweisprachigen Lexikographie. Eine einführende Übersicht über die Forschungs- und Problemlage. Lexikos 10: 32-74.

Wiegand, Herbert Ernst. 2000. Wissen, Wissensrepräsentation und Printwörterbücher. Heid, U., St. Evert, E. Lehmann und Ch. Rohrer (Hrsg.). 2000. Proceedings of the Ninth EURALEX International Congress. EURALEX 2000. Stuttgart, Germany, august 8-12.2000: 15-38. Stuttgart: Institut für Maschinelle Sprachverarbeitung, Universität Stuttgart.

Wiegand, Herbert Ernst. 2002. Adressierung in zweisprachigen Printwörterbüchern. Wiegand, H.E. (Hrsg.). 2002. Studien zur zweisprachigen Lexikographie mit Deutsch VIII: 111-175. Germanistische Linguistik 166. Hildesheim/Zürich/New York: Georg Olms Verlag.

Wiegand, Herbert Ernst. 2005. Angaben, funktionale Angabezusätze, Angabetexte, Angabestrukturen, Strukturanzeiger, Kommentare und mehr. Ein Beitrag zur Theorie der Wörterbuchform. Lexicographica 21: 202-379.

Wiegand, Herbert Ernst. 2007. Il riferimento indirizzato nei dizionari bilingui a stampa. Studi Italiani di Linguistica Teorica e Applicata 36: 7-87.

Wiegand, Herbert Ernst. 2007a. Adressierung in Printwörterbüchern. Präzisierungen und weiterführende Überlegungen. Lexicographica 22: 187-261.

Wiegand, Herbert Ernst. 2010. Hybride textuelle Strukturen und hybride textuelle Einheiten. Ein Beitrag zur Theorie der Wörterbuchform. Lexikos 20: 495-539.

Wiegand, Herbert Ernst. 2011. Adressierung in der ein- und zweisprachigen Lexikographie. Eine zusammenfassende Darstellung. Kürschner, W. (Hrsg.). 2011. Miscellanea Linguistica. Arbeiten 
zur Sprachwissenschaft: 109-234. Frankfurt a.M. [u.a.]: Peter Lang.

Wiegand, Herbert Ernst. 2014. Kotextspezifische Semantik, Pragmatik und Wörterbuchform. Lexicographica 30: 379-487.

Wiegand, Herbert Ernst. 2015. Funktionale Angabezusätze und hybride textuelle Strukturen in Wörterbuchartikeln. Korrigierende Ergänzungen, weiterführende Vertiefungen, zusammenfassende Übersichten und mehr. Lexicographica 31: 249-329.

Wiegand, Herbert Ernst und Rufus H. Gouws. 2013. Addressing and Addressing Structures in Printed Dictionaries. Gouws, R.H., U. Heid, W. Schweickard, H.E. Wiegand (Hrsg.). 2013: 273-314 